Gut, 1978, 19, 1090-1098

\title{
Re-evaluation of the techique of organ culture for studying gluten toxicity in coeliac disease ${ }^{1}$
}

\author{
H. P. HAURI, M. KEDINGER, K. HAFFEN ${ }^{2}$, H. GAZE, B. HADORN, AND \\ W. HEKKENS
}

With the technical assistance of U. Luginbühl

From the Gastrointestinal Unit, Department of Paediatrics, University of Bern, Bern, Switzerland, Unité de Recherche de Physiopathologie Digestive, INSERM Unité 61, Strasbourg-Hautepierre, France, and the Department of Gastroenterology, University Hospital, Leyden, The Netherlands

SUMMARY In vitro cytotoxicity of four different gluten fractions was tested in organ culture for up to 48 hours using flat intestinal biopsies from children with coeliac disease. The fractions were (1) a peptic-tryptic digest of gliadin containing a moderate amount of alpha-gliadin, (2) a peptic-tryptic digest of gluten (Frazer fraction III) from a strain of wheat with a high content of alpha-gliadin, (3) alpha-gliadin, and (4) alpha-GT-18000, a tryptic fragment of alpha-gliadin. The latter three fractions were toxic to coeliac patients in vivo. In vitro, however, none of these fractions proved to be cytotoxic. When added to the culture medium they were not capable of inhibiting the regeneration of the surface epithelium as visualised by histology and electron microscopy. The only difference between cultures with and without gluten fractions was that the former produced slightly more mucus when maintained in vitro as observed in the dissecting microscope. Furthermore, for Frazer fraction III the absence of apparent toxicity was confirmed by the behaviour of brush border enzyme activities during culture. Our results are not in accordance with those reported in the literature. We believe that the criteria used at the present time for the assessment of gluten toxicity in vitro should be extended to include the process of enterocyte desquamation.

Since 1969 it has been possible to use the technique of organ culture as an in vitro tool for the study of the physiology and pathophysiology of the mucosa of the human small intestine (Browning and Trier, 1969; Trier, 1976). Trier and Browning (1970), using biopsies from adult patients with active coeliac disease, showed by electron microscopy that the surface epithelium was capable of regeneration when cultured for 24 hours in vitro. Similar findings have been reported for biopsies from children with untreated coeliac disease (Townley et al., 1973; Jos et al., 1974; Kedinger et al., 1974; Ritis and Jos, 1974). The in vitro normalisation of the mucosa was accompanied by an increase in alkaline phosphatase activity (Falchuk et al., 1974). The regeneration of the

\footnotetext{
${ }^{1}$ Part of this work was reported at the 3rd International Coeliac Symposium, Galway, Ireland, September 1977. ${ }^{2}$ Address for correspondence: Dr K. Haffen, Unité INSERM 61, 3, Avenue Molière, 67200 Strasbourg, France.
}

Received for publication 3 July 1978 biopsies was inhibited by various fractions of peptictryptic digested gliadin (Townley et al., 1973; Falchuk et al., 1974; Jos et al., 1974; Jos et al., 1975).

The question arises, however, of whether the toxicity of gliadin preparations in organ culture can be directly compared with their toxic action in vivo, as cytotoxicity in vitro was not limited exclusively to coeliac mucosa (Townley et al., 1973) and hydrolysed casein was also toxic (Jos et al., 1974, 1975). The present study was undertaken to elucidate further the mechanism of gliadin toxicity in organ culture. We report the absence of in vitro enterotoxicity of various gluten fractions that proved to be toxic to coeliac patients in vivo.

\section{Methods}

The diagnosis of coeliac disease was established according to the criteria of Visakorpi (1974)—-that is, (1) a structurally abnormal intestinal mucosa on a 
gluten-containing diet; (2) a clear improvement of villous structure on a gluten-free diet; (3) deterioration of the mucosa during gluten challenge. Flat biopsies from 15 children who had met criteria (1) and (2) and of eight children who had met all three criteria were included in this study .

In order to establish the persistence of the disease, patients in remission after taking a strict gluten-free diet for at least two years were challenged with raw gluten (Aleuronat, Blattmann and Co., Wädenswil, Switzerland, $0 \cdot 3 \mathrm{~g}$ per $\mathrm{kg}$ body weight per day) for 30 days. Biopsies were taken before and after challenge and compared. The in vivo toxicity of Fluka-gliadin (Fluka, Buchs, Switzerland) was tested in two children with established coeliac disease after complete remission of the mucosa by applying the same concentration over the same period as for the raw gluten. One of these patients showed a severely damaged mucosa after challenge, whereas the mucosa of the other one appeared to be normal under the dissecting microscope and in histology. This patient showed a severely damaged mucosa after two years on normal gluten-containing food.

Biopsies (10-30 mg wet weight) were taken after an overnight fast with a paediatric-sized Watson capsule at the ligament of Treitz and were transferred into the culture medium immediately after excision.

One quarter of the biopsy was fixed in $10 \%$ formaldehyde, photographed and processed for clinical routine histology. Simultaneously, the remainder of the biopsy was cut into three to six pieces. One of these fragments was fixed immediately for electron microscopy, whereas the remaining pieces were maintained in culture with or without one of the following preparations of gliadin or gluten.

\section{GLIADIN OR GLUTEN PREPARATIONS}

\section{PT-gliadin}

Peptic-tryptic digested gliadin (Fluka, Buchs, Switzerland) was hydrolised according to Bronstein et al. (1966) as modified by Cornell and Townley, (1973). The final digest was adjusted to $\mathrm{pH} 7 \cdot 2$ with $\mathrm{HCl}$ and centrifuged for 20 minutes at $2000 \times \mathrm{g}$. The supernatant was immersed into a boiling water bath for 15 minutes to destroy trypsin activity. Trypsin activity was assayed by the method of Hummel (1959) using p-toluenesulfonyl-l-arginine methyl ester as substrate.

The slight precipitate formed during heat inactivation was removed by an additional centrifugation $(20$ minutes at $2000 \times g)$ and the resulting supernatant was lyophilised. PT-gliadin was added to the culture medium in concentrations of 1.0 or $2.0 \mathrm{mg} / \mathrm{ml}$.

\section{Frazer fraction III}

This peptic-tryptic digest of gluten, prepared from the wheat variety Scout 66 following exactly the procedure of Frazer et al. (1959), was heat inactivated (five hours at $70^{\circ} \mathrm{C}$ ).

\section{Alpha-gliadin}

This was prepared from wheat Scout 66. Details of its preparation were given in Eterman et al. (1977).

\section{Alpha-GT-18 000}

This tryptic fragment of alpha-gliadin was prepared as previously described (Hekkens et al., 1974). Residual trypsin activity was inhibited by soy-bean trypsin inhibitor (Sigma).

Organ culture was perfomed in RPMI 1640 medium containing $10 \%$ heat inactivated fetal calf serum and antibiotics as previously described (Hauri et al., 1975). The medium was renewed after 24 hours when the culture was extended to 48 hours. In some cases mucosal specimens were cultured without serum for up to 24 hours. After culture, mucus was thoroughly removed under the dissecting microscope and the explants were rinsed twice with fresh culture medium before morphological analysis or enzymatic determinations.

Each fragment was cut entirely in a series of $1 \mu \mathrm{m}$ thick sections and of ultrathin sections alternately in order to obtain a reliable overall impression. The $1 \mu \mathrm{m}$ sections were stained with toluidine blue and analysed by optical microscopy. Ultrathin sections were stained with uranyl acetate and lead citrate. The morphological analysis was performed by two scientists who did not know if the specimens had been cultured in the presence or absence of gluten fractions.

Protein was determined according to Lowry et al. (1951). Brush border glycosidases were assayed according to Messer and Dahlqvist (1966), and alkaline phosphatase according to Garen and Levinthal (1960) with p-nitro-phenyl-phosphate as substrate. Lactase was measured in the presence of p-chloro-mercuri-benzoate (Asp and Dahlqvist, 1972).

\section{Results}

Non-cultured specimens always lacked villi, had increased intraepithelial lymphocyte counts, and showed elongated crypts. The severity of enterocyte cell damage, however, varied from one individual to another, although all patients obtained a normal gluten-containing food (or extra gluten in the cases 
of challenges) on the days before biopsy. Many biopsies showed large areas of severely injured enterocytes and many had vacuolated cytoplasm (Fig. 1). In such biopsies only the epithelium lining the crypts appeared to be well preserved (Fig. 1a). Almost all the damaged cells exhibited a considerably altered ultrastructure (Fig. 2). Epithelial cells of other biopsies, although cuboidal or less columnar than normal, appeared to be only slightly affected in optical histology (Fig. 3a). However, examination with the electron microscope showed that these cells
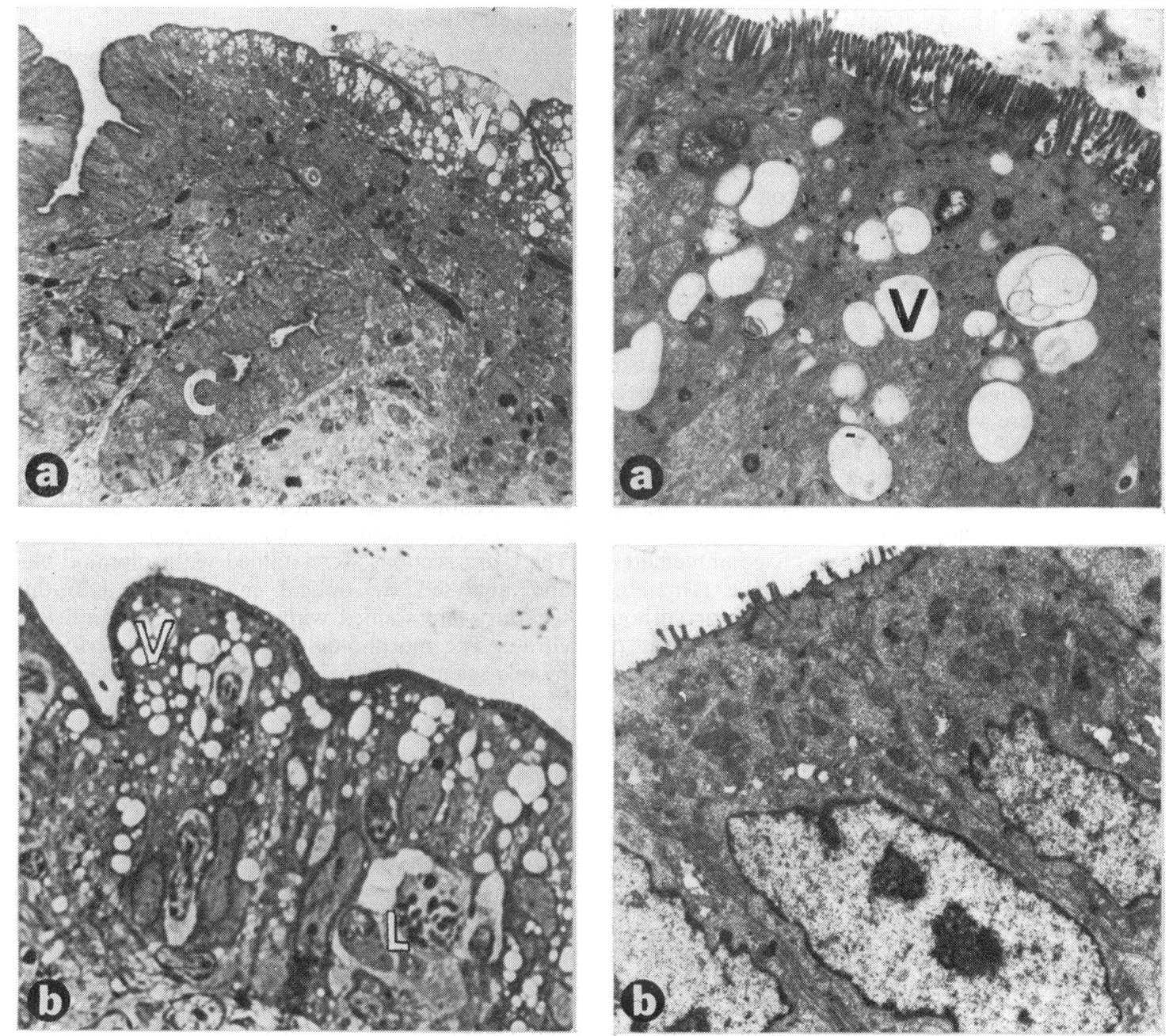

Fig. 1 contained at least some intracellular vacuoles and an increased number of apical lysosomes as well as a subnormal brush border (Fig. 3b).

After 24 or 48 hours of culture in normal medium the biopsy fragments examined under the dissecting microscope were covered by mucus and cellular debris which were carefully removed before the fixation of the tissue. Less damaged biopsies at excision remained histologically unchanged during culture, whereas severely injured ones underwent a remarkable regeneration (Fig. 4) but remained flat.

Fig. 1 Non-cultured biopsy. Histological aspect of a severely damaged epithelium. Toluidine blue staining. $V$ : vacuolated surface cells; $C$ : healthy crypts; L: numerous intraepthelial lymphocytes. $\times 310$ and $\times 1550$.

Fig. 2 Non-cultured biopsies. Electron micrographs illustrating two aspects of the damaged epithelial cells. Subnormal brush border (a) or almost complete absence of microvilli $(b)$ can be observed. $V$ : intracytoplasmic vacuoles. $\times 6110$ and $\times 5520$. 

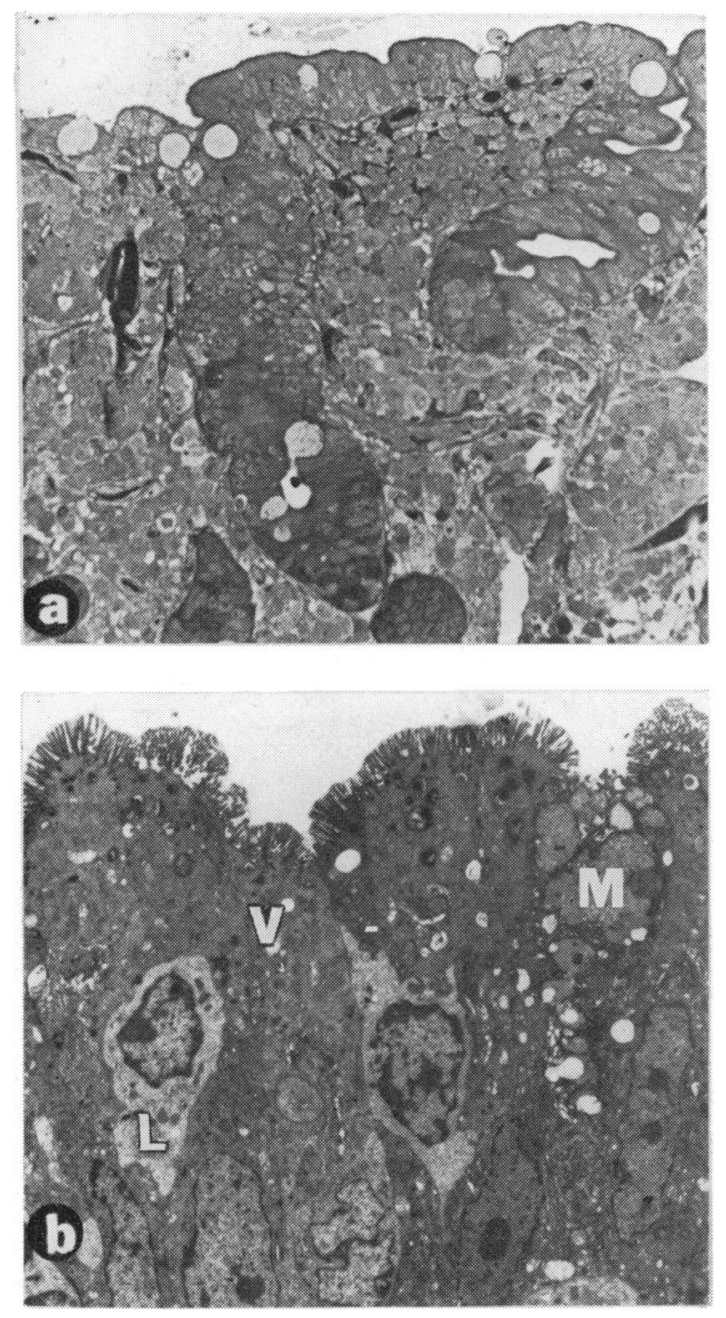

Fig. 3 Light (a) and electron (b) micrographs of a non-cultured biopsy specimen showing a largely undamaged flat epithelium. $M$ : mucus cell; $V$ : intracytoplasmic vacuoles; L: lymphocyte. $\times 340$ and $\times 3170$.

Ultrastructural examination after culture showed that many enterocytes were cylindrical with a welldeveloped, in some cases almost normal, brush border (Fig. 6a). The filaments of the microvillous core had prominent roots in the terminal web. In most cases a depletion of lymphocytes from the lamina propria was observed, but some intraepithelial lymphocytes were still visible. In some cases small areas with heavily damaged enterocytes were still present (not shown), which suggests that not all damaged cells had been sloughed off during culture. Surprisingly, none of our tested gluten fractions proved to be cytotoxic to the surface epithelium in culture (Table). The same histological improvement of the epithelial cells was observed (Fig. 5) as in the control fragments cultured without gluten (Fig. 4), and even on the ultrastructural level no signs of toxicity were found (Fig. 6). Whenever more than one fragment from one biopsy was cultured in the presence of a particular gluten fraction, the histological aspect was essentially the same. The in vivo picture of injured surface enterocytes but predominantly intact crypt cells has never been observed after culture in the presence of gluten fractions. In the rare cases with necrosis after culture both the surface and crypt cells were affected and necrosis was also present in the control specimen cultured without gluten (Table). It has to be pointed out that all micrographs and electron micrographs given in this study were taken from patients in whom the persistence of gluten sensitivity has been subsequently established.

Furthermore, the possible toxicity of Frazer fraction III towards flat biopsies was tested by measuring brush border enzyme activities before and after a 24 hour culture. The behaviour of these enzymatic activities was not significantly different in the presence or absence of this fraction (Fig. 7), confirming the morphological results described above. The only consistent difference between cultures with and without gliadin was the observation in the dissecting microscope that the former produced slightly more mucus than the latter.

\section{Discussion}

The absence of apparent in vitro cytotoxicity from all the tested gluten fractions was surprising, as it does not correspond with the observations reported by Townley et al. (1973), Falchuk et al. (1974), and Jos et al. (1975). We suggest that the difference may be due to (1) the choice of patients, (2) the culture conditions, (3) the fractions used, or (4) the criteria selected to assess toxicity.

In eight out of 23 patients included in our study the persistence of gluten intolerance was established, whereas all other patients showed at least the typical clinical signs of active coeliac disease, had a flat initial biopsy, and responded to a gluten-free diet. The likelihood that patients of the latter group will suffer from permanent gluten sensitivity is $85 \%$ according to our experience based on 45 cases. It is very probable therefore that most of our patients have true coeliac disease.

The conditions for organ culture applied in the present study are comparable with the ones reported by Falchuk et al. (1974). We used the same culture technique (Browning and Trier, 1969) and essentially the same medium (Hauri et al., 1975). As the fetal 

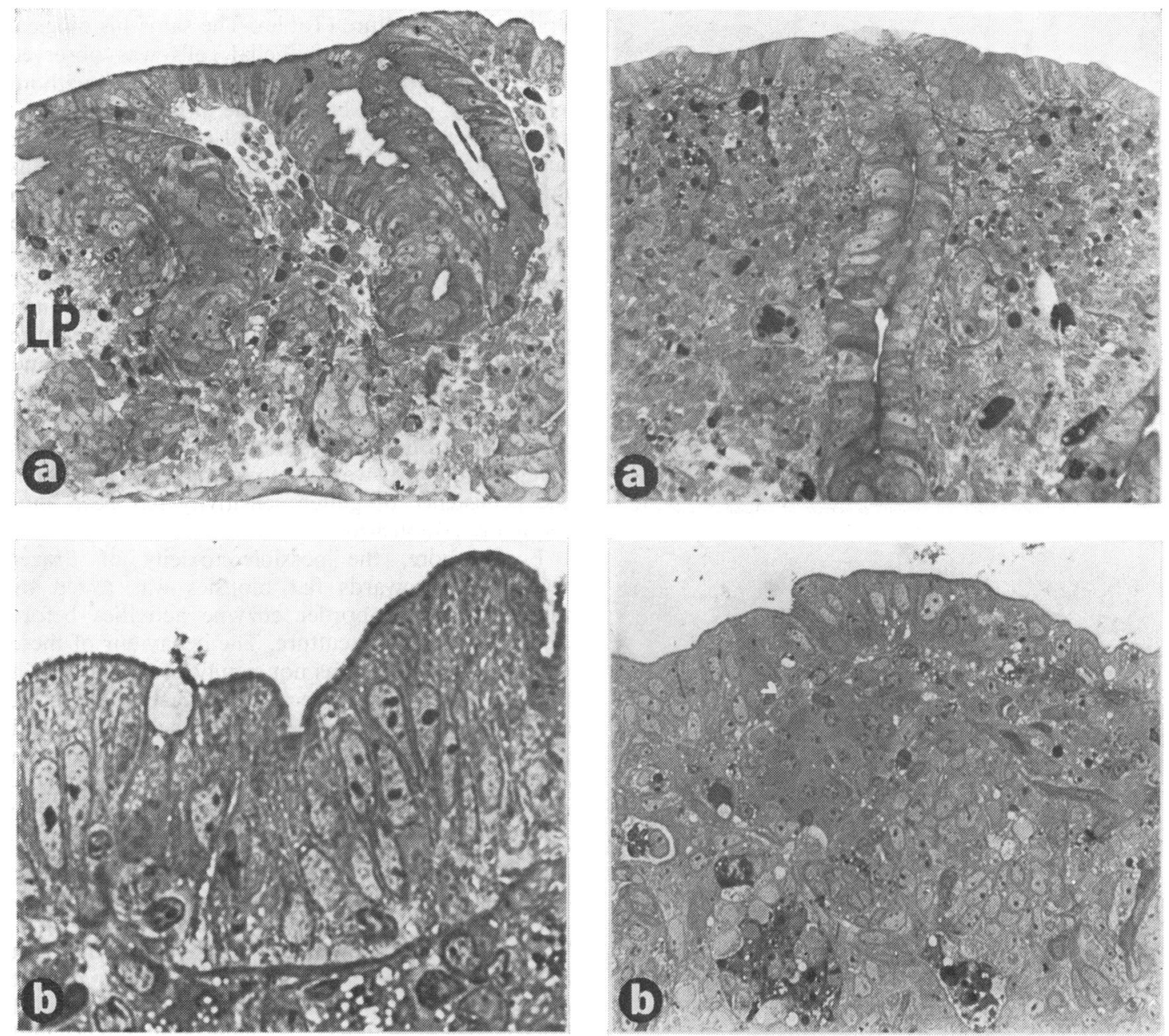

Fig. 4

Fig. 4 Biopsy specimens cultured on control medium for $24(a)$ and 48 hours $(b)$. Note the disappearance of severely damaged epithelial cells shown in Fig. 1. The empty spaces correspond to disappearance of elements of the lamina propria $(L P)$ and are often observed in cultured explants. $\times 340$ and $\times 280$.

Fig. 5 Biopsy specimens cultured on medium containing $2 \mathrm{mg} / \mathrm{ml}$ PT-gliadin for 24 hours (a) and 48 hours $(b, c)$. The histological improvement is comparable with that illustrated in Fig. 4. Note that the specimen shown in (a) originated from the same biopsy as that in Fig. 4a and those shown in (b) and (c) from the same biopsy as that in Fig. 4b. (c) High power micrograph of the surface epithelium illustrated in (b). $\times 300, \times 580$, and $\times 1450$.

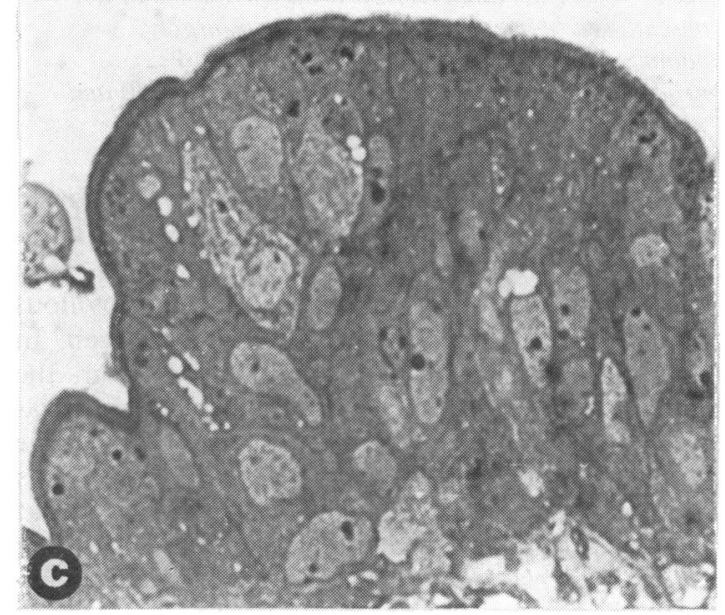

Fig. 5 
Table Behaviour in organ culture of flat intestinal biopsies from 20 children with acute coeliac disease or with coeliac disease in remission after subsequent gluten challenge of 30 days

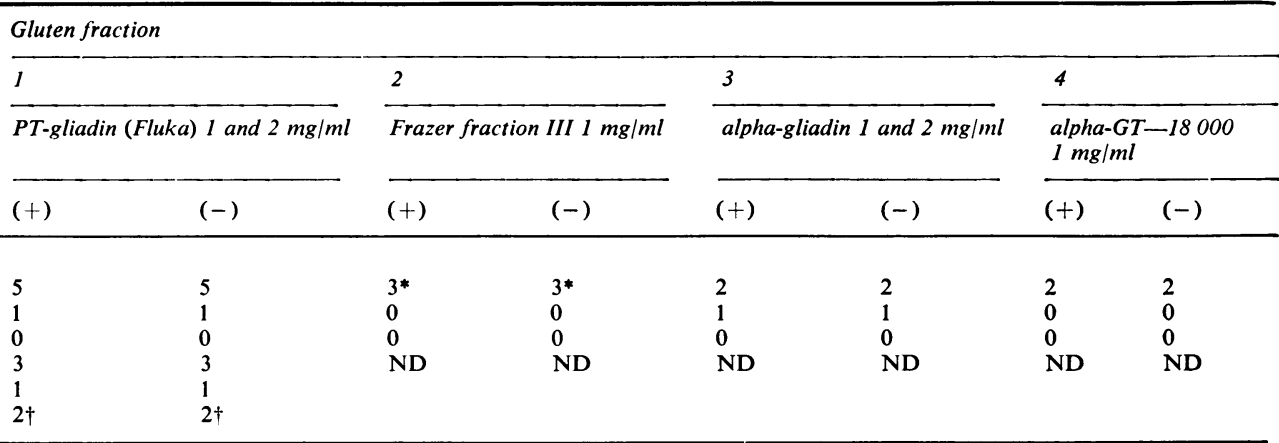

*Cultures without fetal calf serum. †Unspecific necrosis in both the culture with PT-gliadin and the control. ND: not done.

'Regeneration', 'unchanged' or 'damage' respectively characterises the appearance of the epithelial ultrastructure after culture with ( + ) or without ( - control) gluten fractions when compared with the non-cultured fragment of the same biopsy. In no case was a difference observed between specimens cultured in the presence and absence of the four gluten fractions.

It should be noted that, in at least one patient out of each group, the persistence of gluten sensitivity in vivo has been established (all three criteria of Visakorpi (1974) fulfilled): group 1-three cases; group 2-two cases; group 3-one case; group 4-two cases.

calf serum in our culture medium may have a certain capacity to bind toxic proteins (Hudson et al., 1976) we omitted the serum in some instances. Under these conditions, a good survival and regeneration of the surface epithelium was obtained after 24 hours, although the biosynthesis of brush border membrane proteins was markedly reduced compared with cultures with serum (Hauri, unpublished). However, no signs of cytotoxicity could be found with Frazer fraction III under these conditions, which indicates that fetal calf serum was not responsible for the lack of toxic action. Jos et al. (1975) suggested that a culture time of 24 hours may not be sufficient for the detection of cell damage evoked by gliadin, as they obtained cell necrosis only after 48 hours. In our experimental conditions, however, extending the culture period up to 48 hours did not alter the results that were obtained after 24 hours, but showed that areas of non-specific necrosis were more frequent than after 24 hours, regardless of the presence or absence of PT-gliadin. This may, in part, be due to the almost complete absence in suction biopsies of muscularis mucosae whose presence favours tissue survival in culture (Hauri et al., 1975).

As, for ethical reasons, we could test only the undigested gliadin in vivo and not PT-gliadin itself a direct in vivo-in vitro comparison was not possible for this particular fraction. We therefore extended our study to gluten fractions with proven in vivo toxicity. Alpha-gliadin has previously been reported by one of us (Hekkens et al., 1970) to be toxic to patients with coeliac disease. The absence of its cytotoxicity in organ culture may be explained by the poor solubility of this fraction in the medium. More difficult to explain is the absence of in vitro toxicity of alpha-GT-18 000, a soluble tryptic fragment of alpha-gliadin and of Frazer fraction III both of which were toxic in vivo within hours (Hekkens et al., 1974; Hekkens, unpublished). These fractions were used in similar concentrations as those of Falchuk et al. (1974), which were at least twice as high as the minimal concentration reported to give a response in vitro (Jos et al., 1974; Katz et al., 1976).

It is evident from Figs 1,2 , and 3 that noncultured specimens varied not only in the degree of surface cell damage from patient to patient but also within one and the same biopsy. In contrast, the crypt epithelium was always well preserved, confirming the results of Iancu and Elian (1976). We suggest that this variation, which largely concerned the number of cytoplasmic vacuoles and the degree of brush border damage, was due to an inherent property of every individual biopsy, as precautions had been taken to avoid preparation artefacts (organelles like endoplasmic reticulum and mitochondria being well preserved), and the conditions for processing the tissue were kept constant throughout the study. For this reason it is indispensable to analyse every biopsy fragment completely, whenever histology should give reliable results. Despite this careful analysis, we were unable to notice any significant morphological difference in the capacity for surface regeneration between specimens cultured with gluten fractions and their direct controls maintained without gluten. Falchuk et al. (1974) have shown that the regeneration of flat biopsies in vitro is accompanied by an increase in brush border enzyme activities, mainly of alkaline phosphatase 

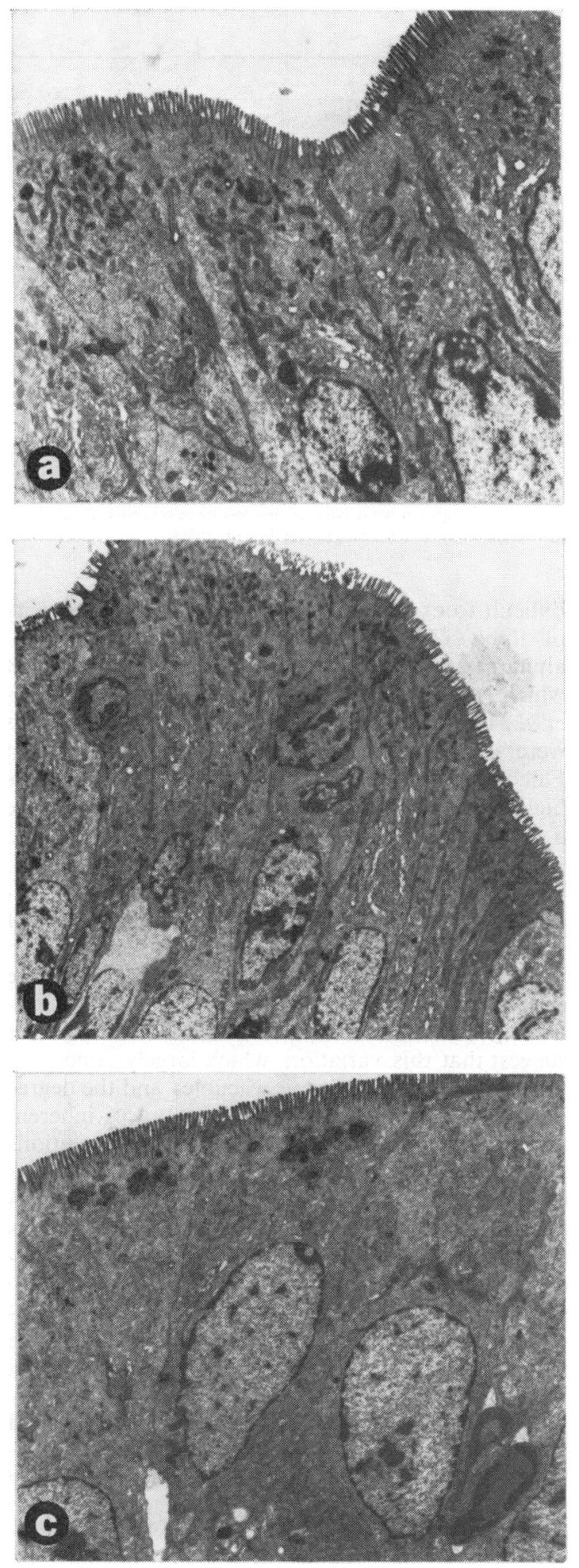

which was inhibited by the presence of gliadin. These results were interpreted as support for the concept that alkaline phosphatase activity may be a direct indicator for tissue regeneration. However, the authors did not state whether or not the mucus layer with the cell-debris was removed before the explants were processed for enzyme measurements. This may be important, as this mucus layer contains a considerable amount of alkaline phosphatase activity (Jos et al., 1974; Hauri et al., 1975; 1977) and bearing in mind our finding that the mucus layer was more pronounced in cultures with gluten fractions. By not removing this sheath, alkaline phosphatase activity does not reflect true tissue regeneration but a sum of enterocyte desquamation and tissue regeneration, By measuring specific alkaline phosphatase of the tissue alone we obtained essentially the same increase regardless of the presence or absence of gluten fractions, which is in accordance with our morphological results.

So far, we can only speculate about the fact that we were unable to find a cytotoxic action by gluten in vitro: in the absence of gluten the severely injured enterocytes of the surface epithelium are replaced by the almost intact upper crypt cells as a result of a more or less normally ongoing process of cell desquamation and epithelial cell migration. In the presence of gluten fractions, however, these processes may be enhanced, leading to a more pronounced mucus-cell debris layer but it is to be expected that the intact enterocytes which had migrated to the surface could better resist gluten toxicity in vitro than in vivo, as the culture medium lacks additional cytotoxic factors, like pancreatic proteases and bile acids, which may enhance tissue destruction initiated by gluten in vivo. This would explain why neither histology nor brush border enzyme activities revealed any significant cytotoxicity. A recent report from Fluge and Asknes (1977) lends support to the above concept. These authors showed that gluten fractions in vitro increased the release of radio-labelled tissue proteins and glyco-proteins from coeliac biopsies and stimulated mitotic activity of crypt cells.

It would appear therefore that histology and the measurement of brush border enzyme activities of the cultured explant alone are insufficient criteria for the assessment of gluten toxicity in vitro and that the

Fig. 6 Representative areas of ultrastructural improvement observed after 24 hours of culture in control medium $(a)$, in serum-free medium containing $1 \mathrm{mg} / \mathrm{ml}$ Frazer fraction III (b), and after 48 hours of culture in medium containing $2 \mathrm{mg} / \mathrm{ml} \mathrm{PT-gliadin}(\mathrm{c})$. $\times 4550, \times 2780$, and $\times 3780$. 


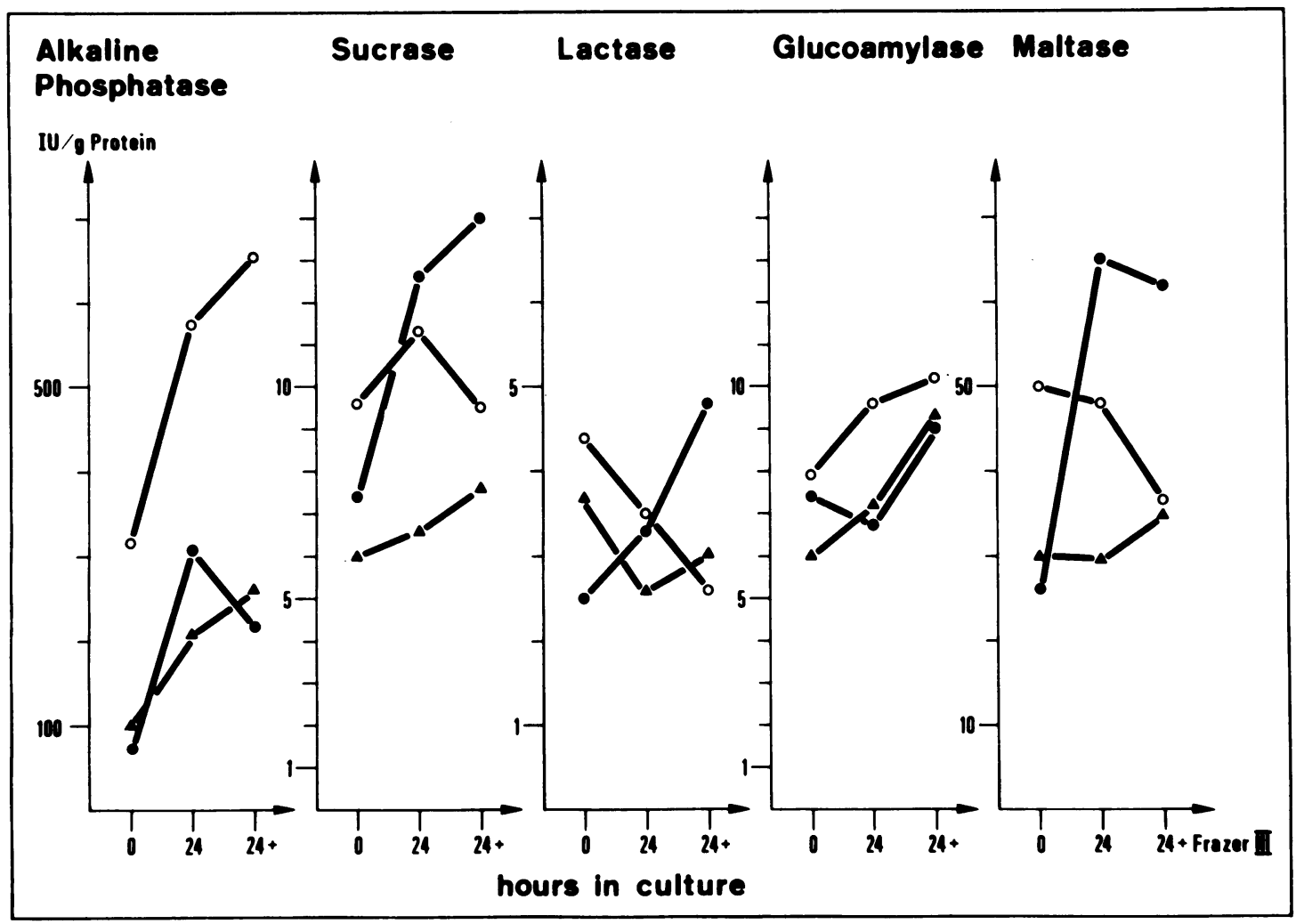

Fig. 7 Brush border enzyme activities in mucosal homogenates of three flat intestinal biopsies before and after a 24 hours' culture in RPMI 1640 with $10 \%$ fetal calf serum. Each biopsy was cut into three fragments, two of which were cultured: one in the presence and one in the absence of $1 \mathrm{mg} / \mathrm{ml}$ of peptic-tryptic digested gluten (Frazer fraction III). It should be noted that all three biopsies used showed the classical histological appearance of coeliac disease and have fulfilled criteria 1 and 2 of Visakorpi (1974).

investigations should be extended to include the process of enterocyte desquamation. Furthermore, it remains to be shown if the in vitro capability of increased epithelial exfoliation is a specific property of gluten.

This work was supported by grants numbers 3.915.72 and 3.676-0.75 from the Swiss National Science Foundation and by grants from the DGRST (No. 737.1630), INSERM, and CNRS (France).

We are grateful to Mrs C. Ryser for secretarial assistance.

\section{References}

Asp, N. G., and Dahlqvist, A. (1972). Human small intestine $\beta$-galactosidase: Specific assay for three different enzymes. Analytical Biochemistry, 47, 527-538.

Bronstein, H. D., Heaffner, L. J., and Kowlessar, O. D. (1966). Enzymatic digestion of gliadin: The effect of the resultant peptides in adult celiac disease. Clinica Chimica Acta, 14, 141-155.
Browning, T. H., and Trier, J. S. (1969). Organ culture of mucosal biopsies of human small intestine. Journal of Clinical Investigation, 48, 1423-1432.

Cornell, H. J., and Townley, R. R. W. (1973). Investigation of possible intestinal peptidase deficiency in coeliac disease. Clinica Chimica Acta, 43, 113-125.

Eterman, K. P., Hekkens, W. T. J. M., Peña, A, S., LemsVan Kan, P. H., and Feltkamp, T. E. W. (1977). Wheat grains: a substrate for the determination of gluten antibodies in serum of gluten-sensitive patients. Journal of Immunological Methods, 14, 85-92.

Falchuk, Z. M., Gebhard, R. L., Sessoms, C., and Strober, W. (1974). An in vitro model of gluten-sensitive enteropathy. Effect of gliadin on intestinal epithelial cells of patients with gluten-sensitive enteropathy in organ culture. Journal of Clinical Investigation, 53, 487-500.

Fluge, G., and Asknes, L. (1977). In vitro Assessment of Gluten Toxicity by Organ Culture of Human Duodenal Mucosa. Third International Coeliac Symposium. Galway, Ireland. MTP Press: Lancaster.

Frazer, A. C., Fletcher, R. F., Ross, C. A. C., Shaw, B., Sammons, H. G., and Schneider, R. (1959). Gluteninduced enteropathy. The effect of partially digested gluten. Lancet, 2, 252-255.

Garen, A., and Levinthal, C. (1960). A fine-structure genetic 
and chemical study of the enzyme alkaline phosphate of E. coli. I. Purification and characterization of alkaline phosphatase. Biochimica Biophysica Acta, 38, 470-483.

Hauri, H. P., Kedinger, M., Haffen, K., Freiburghaus, A., Grenier, J. F., and Hadorn, B. (1977). Biosynthesis of brush border glycoproteins by human small intestinal mucosa in organ culture. Biochmica Biophysica Acta, 467, 327-339.

Hauri, H. P., Kedinger, M., Haffen, K., Grenier, J. F., and Hadorn, B. (1975). Organ culture of human duodenum and jejunum. Biologie et Gastro-entérologie (Paris), 8, 307319.

Hekkens, W. T. J. M., Haex, A. J. C. and Willighagen, R. G. J. (1970). Some aspects of gliadin fractionation and testing by a histochemical method. In Coeliac Disease: Proceedings of an International Conference, pp. 11-19. Edited by C. C. Booth and R. H. Dowling. Churchill Livingstone: Edinburgh.

Hekkens, W. T. J. M., Van den Aarsen, C. J., Gilliams, J. P., Lems-Van Kan, P., and Bouma-Frölich, G. (1974) a-gliadin structure and degradation. In Coeliac Disease: Proceedings of the Second International Coeliac Symposium, pp. 39-45. Edited by W. T. J. M. Hekkens., and A. S. Peña. Stenfert Kroese: Leyden.

Hudson, D. A., Cornell, H. I., Purdham, D. R., and Rolles, C. J. (1976). Non-specific cytoxicity of wheat gliadin components towards cultured human cells. Lancet 1, 339341 .

Hummel, B. C. W. (1959). A modified spectrophotometric determination of chymotrypsin, trypsin and thrombin. Canadian Journal of Biochemistry and Physiology, 37, 1393-1399.

Iancu, T., and Elian, E. (1976). The intestinal microvillus. Ultrastructural variability in coeliac disease and cow's milk intolerance. Acta Paediatrica Scandinavica, 65, 65-73.

Jos, J., Lenoir, G., De Ritis, G., and Rey, J. (1974). In vitro culturing of biopsies from children. In Coeliac Disease: Proceedings of the Second International Coeliac Symposium, pp. 91-105. Edited by W. T. J. M. Hekkens, and
A. S. Peña. Stenfert Kroese: Leyden.

Jos, J., Lenoir, G., De Ritis, G., and Rey, J. (1975). In vitro pathogenetic studies of coeliac disease. Effects of protein digests on coeliac intestinal biopsy specimens maintained in culture for 48 hours. Scandinavian Journal of Gastroenterology, 10, 121-128.

Katz, A. J., Falchuk, Z. M., Strober, W , and Shwachman, H. (1976). Gluten sensitive enteropathy. Inhibition by cortisol of the effect of gluten protein in vitro. New England Journal of Medicine, 295, 131-135.

Kedinger, M., Haffen, K., Hauri, H. P., Schang, J. C., Hadorn, B., and Grenier, J. F. (1974). Organ culture of guinea pig and human intestinal mucosa. Ultrastructural, cytochemical and biochemical study. (Abstract). Digestion, 11, 450-451.

Lowry, O. H., Rosebrough, N. J., Farr, A. L., and Randall, R. J. (1951). Protein measurement with the folin phenol reagent. Journal of Biological Chemistry, 193, 265-275.

Messer, M., and Dahlquist, A. (1966). A one-step ultramicro method for the assay of intestinal disaccharidases. Analytical Biochemistry, 14, 376-392.

Ritis, G. De, and Jos, J. (1974). Organ culture of intestinal mucosa in coeliac disease and in cow's milk intolerance. Effects of protein fractions. (Abstract). Acta Paediatrica Scandinavica, 63, 162.

Townley, R. R. W., Bhathal, P. S., Cornell, H. J., and Mitchell, J. D. (1973). Toxicity of wheat gliadin fractions in coeliac disease. Lancet, 1, 1362-1364.

Trier, J. S. (1976). Organ-culture methods in the study of gastrointestinal-mucosal function and development. New England Journal of Medicine, 295, 150-155.

Trier, J. S., and Browning, T. H. (1970). Epithelial-cell renewal in cultured duodenal biopsies in celiac sprue. New England Journal of Medicine, 283, 1245-125C.

Visakorpi, J. K. (1974). Definition of coeliac disease in children. In Coeliac Disease: Proceedings of the Second International Coeliac Symposium, pp. 10-16. Edited by W. T. J. M. Hekkens and A. S. Peña. Stenfert Kroese: Leyden. 